\title{
Schreiben von Prof. L. Swift betr. Nachforschungen nach dem Brorsen'schen Cometen.
}

This morning I made my fourth and last attempt to find Brorsen's Comet. The seeing was good, and using the 16 in. glass with my comet eye piece giving a power of $13^{2}$, and a field of $3 I^{\prime}$, I searched carefully in the regions of its three computed places and saw nothing of it. Day light cut the search short though it was prolonged a sufficient length of time to convince me that if the comet was there it was equal in faintness to some of my $» e$ e $F$ nebulae .

Have we here the case of another comet lost?

Warner Observatory, Rochester N. Y., I 890 March 31.

Lewis Swift.

\section{Mathematische Preisaufgabe der Fürstlich Jablonowski'schen Gesellschatt zu Leipzig fur das Jahr 1893}

Durch die allgemeinen Untersuchungen von Herrn Lie über die Differentialinvarianten der endlichen und unendlichen Transformationsgruppen*) sind die Mittel und Wege gegeben, um zu einer Invariantentheorie beliebiger Differentialgleichungen zu gelangen. Die betreffenden allgemeinen Methoden von Lie sind in den zahlreichen Untersuchungen über die Invarianten specieller Differentialgleichungen fast gar nicht berücksichtigt worden, es erscheint der Gesellschaft daher wünschenswerth, dass

die Invariantenbestimmung einer ausgedehnteren Categorie zunächst von gewöhnlichen Differentialgleichungen auf Grund der Lie' schen Begriffsbestimmungen und Methoden

in Angriff genommen werde. Um die Art der Aufgaben zu bezeichnen, deren Erledigung der Gesellschaft erwiinscht sein würde, führen wir beispielsweise an die Bestimmung der Invarianten, welche die allgemeine Differentialgleichung zweiter Ordnung

$$
y^{\prime \prime}=\infty\left(x, y, y^{\prime}\right)
$$

einer Ebene $(x, y)$ gegenüber der unendlichen Gruppe

$$
x_{1}=\varphi(x, y), \quad y_{1}=\chi(x, y)
$$

Bestinmung aller Invarianten eines Differentialausdrucks erster Ordnung

gegenüber der genannten unendlichen Gruppe*).

Preis 1000 Mark.

Die anonym einzureichenden Bewerbungsschriften sind, wo nicht die Gesellschaft im besondern Falle ausdrücklich den Gebrauch einer andern Sprache gestattet, in deutscher, lateinischer oder französischer Sprache zu verfassen, müssen deutlich geschrieben und paginirt, ferner mit einem Motto versehen und von einem versiegelten Umschlag begleitet sein, welcher auf der Aussenseite das Motto der Arbeit trägt, inwendig den Namen und Wohnort des Verfassers angiebt. Jede Bewerbungsschrift muss auf dem Titelblatte die Angabe einer Adresse enthalten, an welche die Arbeit für den Fall, dass sie nicht preiswürdig befunden würde, zurückzusenden ist. I) Zeit der Einsendung endet mit dem 30. November des angegebenen Jahres, und die Zusendung ist an den Sekretär der Gesellschaft (für das Jahr 1890 Geh. Hofrath Prof. Dr. Rudolph Leuckart, Thalstr. 33) zu richten. Die Resultate der Prüfung der eingegangenen Schriften werden durch die Leipziger Zeitung im März oder April des folgenden Jahres bekannt gemacht. Die gekrönten Bewerbungsschriften werden Eigenthum der Gesellschaft.

aller Punkttransformationen dieser Ebene besitzt, oder die , Bew

**) (1) und $\boldsymbol{Q}$ sollen hier sogen, janalytischer Functionen bezeichnen, welche in der Umgebung eines Werthsystems $x_{0} y_{0} y_{0}^{\prime}$ von allgemeiner Lage, in gewöhnliche Potenzreihen von $x-x_{0}, y-y_{0}, y^{\prime}-y_{0}^{\prime}$ entwickelt werden können.

\section{Literarische Anzeigen.}

Dr. Rudolf Wolf. Handbuch der Astronomie, ihrer Geschichte und Literatur. In zwei Bänden.

Der eben erschienene erste Halbband umfasst das erste Buch des Werkes: Aufgabe, Geschichte und Vorkenntnisse in sechs Abschnitten. Die folgenden Bücher werden enthalten: Einleitung in die Astronomie, Theorie der Instrumente und Messungen, und Mechanik und Physik des Himmels. Der Leser wird auch bei diesem Werke die grosse Belesenheit des Verfassers in allen Theilen der Astronomie und der ihr verwandten Wissenschaften bewundern.
Dr. Ernst Meissel. Ueber die Bessel'schen Functi. onen $\mathrm{I}_{k}^{0}$ und $\mathrm{I}_{k}^{l}$. Programm der Ober-Realschule in Kiel. 1890 .

Der $\forall$ erfasser giebt in vorliegender kleiner Schrift, im Anschluss an seine Tafeln für die genannten beiden Functionen (vergl. A. N. Nr. 2912 , Bd. I 22 , S. I 59) verschiedene interessante Entwickelungen und behandelt auch die Bessel' schen Functionen mit imaginären Argumenten. Einige Exemplare der Schrift können auf Verlangen noch von mir zuI gesandt werden.
$K r$.

In halt zu Nr. 2965. H. Seeliger. Notiz übcr einen Tisserand'schen Satz, die Umgestaltung der Cometenbahnen betreffend. 209. - K. Schwarzschild. Zur Bahnbestimmung nach Bruns. 21 I. $-K$. Schroaraschild. Methode zur Bahnbestimmung der Doppelsterne. 215. B. Matthiessen. Beobachtungen des Mondes und der Mondsterne. 217. - W. R. Brooks. Nachricht über die Entdeckung des Cometen 1890... (Brooks MIärz 19). 221. - W. Luther. Beobachtung des Cometen I890... (Brooks März I9). 221. L. Swift. Schreiben betr. Nachforschungen nach dem Brorsen'schen Cometen. 223. - Mathematische Preisaufgabe der Fürstlich Jablonowski'schen Gesellschaft zu Leipzig. 223. - Literarische Anzeigen. 223. 